\title{
Guías de atención médica para dejar de fumar. Una propuesta para Latinoamérica
}

\author{
Raúl H Sansores, MSc, (') Oliver Pérez-Bautista, MC Neumól,(') Alejandra Ramírez-Venegas, MSc. (I)
}

\section{Sansores RH, Pérez-Bautista $O$, Ramírez-Venegas A. \\ Guías de atención médica para dejar de fumar. Una propuesta para Latinoamérica. Salud Publica Mex 2010;52 supl 2:S355-S365.}

\section{Resumen}

Objetivo. Aunque la prevalencia del tabaquismo es diferente en cada país de América, los daños a la salud se presentan en la misma proporción. Por ello, es indispensable contar con guías de tratamiento que idealmente sean de utilidad para todos los países. Material y métodos. Para hacer recomendaciones puntuales de utilidad a la región se realizó una búsqueda en PubMed de los últimos cinco años, con los títulos "Smoking cessation guidelines" y "Smoking cessation treatment meta-analysis" para conocer el contenido de las diferentes guías y de los títulos "Nicotine replacement therapy", "Nicotine chewing gum", "Nicotine patches" "Nicotine inhaler", "Bupropion therapy", "Varenicline therapy" e "Individual and grupal behavioural counselling for smoking cessation" para determinar la eficacia de cada intervención. Resultados y conclusión. Nuestra recomendación basada en la disponibilidad de medicamentos y de recursos humanos es que el consejo médico y la terapia conductual grupal deben ser acciones primarias acompañadas, o no, de medicamentos de primera línea.

Palabras clave: guías de tratamiento, tratamiento para dejar de fumar; tabaquismo

\author{
Sansores RH, Pérez-Bautista O, \\ Ramírez-Venegas A. \\ Proposal of Latin-American guidelines \\ for smoking cessation. \\ Salud Publica Mex 2010;52 suppl 2:S355-S365.
}

\begin{abstract}
Objective. Although prevalence of tobacco smoking is different in the Latin American regions, the consequences on health are similar. Material and Methods. Therefore, guidelines on treatment are needed and ideally speaking must be useful in the different countries. In order to make quick recommendations useful in the region we made a search in PubMed from the last 5 years with the titles "Smoking cessation guidelines" and "Smoking cessation treatment meta-analysis" to know the content of different guidelines and with the titles of "Nicotine replacement therapy", "Nicotine chewing gum", "Nicotine patches","Nicotine inhaler","Bupropion therapy", "Varenicline therapy" and "Individual and grupal behavioural counselling for smoking cessation" to determine the efficacy of each intervention. Results and conclusion. Our recommendation based on the availability of pharmacotherapy and human resources is that medical advice and behavioural grupal counseling must be primary interventions either adding, or not, first line drugs for smoking cessation
\end{abstract}

Key words: guidelines for smoking cessation; tobacco smoking

(I) Instituto Nacional de Enfermedades Respiratorias. México DF, México.

Fecha de recibido: 7 de abril de 2010 - Fecha de aceptado: 13 de julio de 2010

Solicitud de sobretiros: M en C. Raúl H Sansores. Departamento de investigación en Tabaquismo y EPOC.

Instituto Nacional de Enfermedades Respiratorias. Calzada de Tlalpan 4502. 14090 México DF.

Correo electrónico: raulsansores@yahoo.com.mx 
E tabaquismo es un síndrome inflamatorio crónico, lentamente progresivo, de daño multisistémico, asociado con la adicción a la nicotina. ${ }^{1}$ La adicción es responsable del creciente número de fumadores. La Organización Mundial de la Salud (OMS) ha estimado que la prevalencia global es de $30 \%$ y que el tabaquismo es la principal causa de muerte prevenible. ${ }^{2}$ En el año 2000, se documentaron 4.8 millones de muertes. ${ }^{3}$ En el continente americano las enfermedades asociadas con el consumo de tabaco fueron responsables de aproximadamente 1 millón de defunciones. Si las tendencias actuales no se revierten, para 2030 cerca de 10 millones de personas morirán en todo el mundo por enfermedades relacionadas con el consumo de tabaco, incluyendo tanto a los fumadores activos como a quienes se exponen de manera involuntaria al humo de los cigarrillos.

La prevalencia del tabaquismo no es igual en todos los países del continente americano. El estudio PLATINO documentó en cinco ciudades de Latinoamérica (San Paulo, Brasil; Ciudad de México, México; Montevideo, Uruguay; Santiago de Chile, Chile; y Caracas, Venezuela), que la prevalencia del tabaquismo en adultos de 40 años fue notablemente mayor en algunas ciudades que en otras. ${ }^{4}$ Por ejemplo, en Chile, Uruguay y Venezuela la prevalencia fue de $38.5,28$ y $28.5 \%$, respectivamente, mientras que en México fue de $25.4 \%$. Latinoamérica fue considerada en la etapa II de la epidemia de tabaquismo. La prevalencia fue mayor en los hombres adultos. Usando un análisis de cohorte se observó que la mayor prevalencia oscila entre los 20-29 años de edad, mientras que la más alta incidencia está entre los 10-19 años de edad. A pesar de estas diferencias, los problemas de salud relacionados con la adicción a los productos del tabaco son similares. Por lo tanto, se requieren estrategias para ayudar a los fumadores a dejar de fumar.

Este trabajo tiene dos objetivos: primero, determinar cuáles son las recomendaciones actualmente prevalentes en el tratamiento del tabaquismo y, segundo, hacer una revisión de la eficacia de las intervenciones actualmente disponibles. Finalmente a partir del análisis de dichos hallazgos, se ofrece una propuesta global, con énfasis en los países latinoamericanos, que considera el aspecto económico de la región.

\section{Material y métodos}

Realizamos una búsqueda en PubMed con las palabras "Smoking cessation Guidelines" y "Smoking cessation Treatment meta-analysis". La búsqueda sólo incluyó lo que se ha publicado de 2005 a la fecha. Solamente consideramos para este trabajo los que en PubMed aparecen como "Reviews" de metaanálisis o como metaánalisis que estuvieran escritos en inglés. En términos gene- rales, no incluimos trabajos originales aislados sobre ningún medicamento o intervención farmacológica o no farmacológica. Particular atención pusimos a las guías de origen hispano (Latinoamérica, incluyendo Brasil y España). Asimismo, hicimos una revisión de las guías publicadas cuando menos en documentos oficiales, aunque no estén dentro de bases científicas como PubMed. Entre éstas, revisamos las guías mexicanas, ${ }^{5}$ así como las de Argentina, Costa Rica, Panamá, Uruguay $^{6}$ y las destinadas a países de escasos recursos económicos (UICTER). ${ }^{7}$ El objetivo de esta revisión fue determinar las recomendaciones farmacológicas y no farmacológicas para el tratamiento del tabaquismo. Además, hicimos una revisión específica para cada área de tratamiento con las siguientes especificaciones: "Nicotine replacement therapy" (NRT), "Nicotine chewing gum", "Nicotine patches", "Nicotine inhaler", "Bupropion therapy, "Varenicline therapy" e "Individual behavioural counselling for smoking cessation both individual as grupal".

Al final, hicimos una propuesta de lo que los países latinoamericanos deben incluir en su tratamiento y cómo debe prepararse la población con base en la disponibilidad real de medicamentos auxiliares para dejar de fumar y de los recursos humanos. Una parte de los resultados y de la discusión se basó en los tratamientos farmacológicos disponibles en México.

\section{Resultados}

Encontramos 68 trabajos con el título "Smoking cessation Guidelines", 34 de ellos publicados de 2005 en adelante, 171 con el título de "Smoking cessation treatment meta-analysis", de los cuales fueron 131 revisiones y sólo 65 aparecieron publicados de 2005 a la fecha. Sólo tres trabajos aparecen como "guías de tratamiento", uno es de Brasil, ${ }^{8}$ otro corresponde a las guías GOLD (Guidelines for Diagnosis of Obstructive Lung Diseases) ${ }^{9} \mathrm{y}$ otro corresponde al uso de las guías. ${ }^{10}$ La mayor parte de las guías existentes no están publicadas en este periodo, pero Raw y colaboradores las resumieron en un excelente trabajo. ${ }^{10}$ De acuerdo con su trabajo, de 31 países participantes, $81 \%$ tiene guías y de los cuatro países latinoamericanos encuestados (Brasil, Chile, México y Argentina), todos reportaron tener guías para el manejo del tabaquismo. Sin embargo, sólo 19\% de los países encuestados, reportó contar con un sistema nacional de tratamiento y de los países latinos, ninguno. En términos generales, todas proponen el abordaje médico, el abordaje conductual y las diferentes intervenciones farmacológicas pero solamente en $47 \%$ de los países encuestados hay una persona responsable, en $24 \%$ se consigue ayuda fácilmente con un médico general y en 19\% en un centro especial para 
adicciones. Desde el punto de vista del tratamiento farmacológico, en más de 90\% de los países encuestados se consigue terapia de reemplazo de nicotina, en $75 \%$ bupropión, y en $69 \%$, varenicline. En los cuatro países latinos sí hay la disponibilidad farmacológica.

Los resultados de las propuestas de tratamiento se pueden dividir, de acuerdo a los resultados de nuestra búsqueda, en farmacológicas y no farmacológicas. En las primeras abordaremos solamente aquellas que tienen aprobación de la Food and Drug Administration de Estados Unidos de América (FDA, por sus siglas en inglés) y que están dentro de las propuestas de las diferentes guías y revisiones que encontramos. Las discutiremos brevemente, comenzando con aquellas que han reportado su probabilidad de éxito en el proceso de dejar de fumar.

\section{Terapia farmacología}

\section{Terapia de reemplazo de nicotina (TRN)}

Encontramos 448 revisiones en PubMed con la búsqueda específica de "Replacement nicotine therapy". Solamente 204 se publicaron de 2005 en adelante. La terapia de reemplazo de nicotina puede administrarse en forma de goma de mascar, parches, inhalador oral, aerosol nasal y una forma relativamente nueva, conocida en inglés como lozenge. La tasa de abstinencia a 6 y 12 meses, tanto de la goma de mascar como de los parches se puede observar en el cuadro I. En las guías de Fiore ${ }^{11}$ se reporta que el uso del aerosol nasal está asociado con un éxito elevado para dejar de fumar (RM 2.3; IC95\%; 1.7-3.0). Cifras similares reporta para el inhalador de nicotina (RM 2.1; IC95\%; 1.5-2.9). Sin embargo, aunque el inhalador está disponible en México, comercialmente hablando, no existe esta disponibilidad para la mayoría de los países latinoamericanos. Es por ello que la discusión la centraremos sobre la goma de mascar, los parches y el inhalador.

Goma de mascar. La eficacia de esta presentación de TRN se resume en el cuadro I, el resultado de 3 metaanálisis. Es de notar que en el de Moore y colaboradores ${ }^{12}$ la RM es de 1 y que el promedio de los tres es igual a 1.61. Viene en forma de tableta de resina de nicotina que le permite ser masticable y que puede contener de 2 a 4 $\mathrm{mg}$ de nicotina. La nicotina se absorbe totalmente por la mucosa oral. En algunos países como México hay una presentación con sabor menta para estimular al fumador a usar el medicamento como chicle con un sabor más agradable que la tableta tradicional. En la mayor parte de los países latinos no está disponible la presentación con sabor menta. El número de tabletas que se recomiendan depende del grado de adicción y ésta se puede determinar utilizando el cuestionario de Fagerstrom. Se recomienda un máximo de 12 tabletas al día y pueden ser menos si la tableta se divide en mitades, incluso cuartos, porque la cantidad total de nicotina que se absorbe depende de la frecuencia y el vigor de cada masticada. Nosotros recomendamos dividirla y después de extraer nicotina con unas cuantas masticadas, guardar la goma entre los molares y la encía y volver a masticarla cuando se presente el deseo de fumar. Una vez que el fumador ha disminuido en forma paulatina el número de cigarros al día, es recomendable disminuir también, en forma progresiva, el número de tabletas de nicotina que consume al día.

Parches de nicotina. Hay cuando menos dos presentaciones que se diferencian por el tiempo que liberan nicotina y por lo tanto de la cantidad total de nicotina que se libera. La nicotina que se libera en el día depende de la superficie total de cada parche y la cantidad de nicotina contenida en cada parche $\left(5 \mathrm{mg} / \mathrm{cm}^{2}\right.$, por ejemplo). Los parches que liberan nicotina durante 24 horas vienen en tres presentaciones diferentes, cuya superficie total $\left(7,15\right.$ y $\left.22 \mathrm{~cm}^{2}\right)$ depende la cantidad de nicotina que se libera en el día en forma constante y que en total corresponde a 7, 14 y $21 \mathrm{mg}$ en 24 horas. Los parches que se usan durante 16 horas liberan en total 5, 10 y 15 $\mathrm{mg}$, respectivamente. La decisión de recomendar unos $\mathrm{u}$ otros depende de preferencias personales o de que los pacientes refieran insomnio con el de 24 horas. La eficacia de los parches como auxiliares para dejar de fumar está resumida en el cuadro I.

Inhalador de nicotina. Encontramos 65 revisiones en PubMed con la búsqueda específica de "Nicotine inhaler". Sólo 25 de ellas aparecen publicadas en los últimos cinco años. Dos que estaban escritas en alemán, una en polaco y otra en italiano, las eliminamos de nuestra revisión. El resumen de su eficacia se puede observar en el cuadro I. El metaanálisis de Moore y colaboradores ${ }^{12}$ es particularmente interesante, porque incluyeron solamente fumadores que no tuvieran deseos de dejar de fumar y que hayan conseguido dejar de fumar por seis meses. Este último objetivo lo consiguieron haciendo una estimación probabilística. Ellos solamente encontraron dos ensayos clínicos controlados.

\section{Bupropión}

El bupropión es un antidepresivo, y aunque existen otros, como la nortriptilina que ha mostrado buenos resultados para ayudar a los fumadores a dejar de fumar, el único recomendado por la FDA como auxiliar 
para dejar de fumar es el bupropión. De hecho, fue el primer medicamento no nicotínico que formalmente fue aprobado por la FDA, después que se observó que sus efectos antidepresivos se acompañaban de intentos no programados para dejar de fumar. Desde entonces se han publicado una cantidad importante de evidencias que señalan su eficacia como auxiliar para dejar de fumar. Sin embargo, de 2005 a la fecha encontramos 66 trabajos y solamente en tres ${ }^{13-15}$ aparece analizado dentro de un grupo de medicamentos, incluyendo una comparación directa con varenicline y solamente uno se ocupa exclusivamente de bupropión en cuanto a sus efectos secundarios. ${ }^{16}$ En el cuadro II se muestra un resumen de ello. Su mecanismo de acción consiste fundamentalmente en la inhibición de la recaptura de dopamina y noradrenalina, ${ }^{17}$ dando como resultado el incremento en el bienestar general del paciente. El bupropión está disponible en más de 70\% de los países en donde hay programas para dejar de fumar, pero la mayor parte de los sistemas de salud para la población no lo ofrecen sin costo, es decir, el fumador debe pagar. ${ }^{10}$ Del bupropión se recomienda 150 mg vía oral dos veces al día. Es recomendable fijar una fecha para dejar de fumar cuando menos dos semanas después de iniciar el tratamiento para permitir que el efecto del medicamento empiece a ser notable. En nuestra clínica indicamos que el tratamiento se prolongue por lo menos nueve semanas, pero en algunos fumadores el tiempo puede ser mayor bajo vigilancia. Debido a que el insomnio es el efecto adverso más frecuente, según se observó en una muestra de más de 11000 sujetos, ${ }^{18}$ sugerimos que la primera dosis se tome a las 8:00 horas y la segunda a las 16:00 horas, para prevenir insomnio en algunos fumadores sensibles.

\section{Cuadro I \\ MetaAnÁlisis de terapia de ReEMPLAZO CON NICOTINA}

\begin{tabular}{lccccc} 
Dispositivo* & Número de estudios & Referencia & Tiempo de evaluación & RM & IC 95\% \\
& 6 & Stead $^{44}$ & 6 meses & 2.20 & $1.50-3.20$ \\
Chicle & 4 & Moore $^{12}$ & 6 meses & 1.00 & $0.36-2.80$ \\
& 22 & Eisenberg $^{14}$ & 12 meses & 1.65 & $1.37-2.01$ \\
\hline \multirow{2}{*}{ Parche } & 32 & Stead $^{44}$ & 12 meses & 1.90 & $1.70-2.20$ \\
& 30 & Eisenberg $^{14}$ & 12 meses & 1.88 & $1.60-2.22$ \\
\hline \multirow{2}{*}{ Aerosol inhalado } & 6 & Stead & 6 meses & 2.10 & $1.50-2.90$ \\
& 1 & Moore & 6 meses & 2.06 & $1.34-3.15$ \\
\hline
\end{tabular}

* Disponibles en México

RM: Razón de momios estimado versus placebo

IC: Intervalo de confianza.

Se muestran 2 o 3 metaanálisis diferentes para cada variedad de terapia de reemplazo con nicotina (TRN). Aunque se puede ver en forma individual los RM por tipo de tratamiento, se hizo un promedio del total de RM de los tres tipos de TRN.

\section{Cuadro II}

MetaAnálisis de Medicamentos no nicotínicos de primera línea

\begin{tabular}{|c|c|c|c|c|c|}
\hline Medicamento & Número de estudios & Referencia & Tiempo de evaluación & $R M$ & IC $95 \%$ \\
\hline Bupropión & $\begin{array}{l}26 \\
16 \\
14\end{array}$ & $\begin{array}{l}\text { Hughes }^{13} \\
\text { Eisenberg' } \\
\text { Wu }^{15}\end{array}$ & $\begin{array}{l}12 \text { meses } \\
12 \text { meses } \\
12 \text { meses }\end{array}$ & $\begin{array}{l}2.00 \\
2.07 \\
1.76\end{array}$ & $\begin{array}{l}1.80-2.20 \\
1.73-2.55 \\
1.10-2.21\end{array}$ \\
\hline & & & Promedio total & 1.94 & \\
\hline Varenicline & $\begin{array}{c}7 \\
13\end{array}$ & $\begin{array}{c}\text { Cahill }^{20} \\
\text { Eisenberg }\end{array}$ & $\begin{array}{l}12 \text { meses } \\
12 \text { meses }\end{array}$ & $\begin{array}{l}2.33 \\
2.41\end{array}$ & $\begin{array}{l}1.95-2.80 \\
1.91-3.12\end{array}$ \\
\hline
\end{tabular}

RM: Razón de momios estimado versus placebo

IC: Intervalo de confianza. Se muestran las RM para cada tratamiento de 3 o 2 metaanálisis diferentes. Abajo de las RM de bupropión y de varenicline se hizo un promedio de las RM de los metaanálisis 


\section{Varenicline}

Después de casi 10 años de haber aparecido bupropión como único medicamento no nicotínico, apareció varenicline en el mercado internacional como medicamento auxiliar para dejar de fumar. Varenicline es un agonista parcial de los receptores nicotínicos de acetilcolina $\alpha 4 \beta 2$. Su mecanismo de acción es único entre los medicamentos actualmente existentes y su eficacia parece ser la mejor. Encontramos 15 trabajos con el título de "Varenicline meta-analysis". Sólo dos analizan exclusivamente varenicline. ${ }^{19-20}$ En otro trabajo que no tiene el perfil preciso de metaanálisis pero que recogió y analizó toda la evidencia posible de ensayos clínicos controlados, metaanálisis, resúmenes de congresos, reporte de casos y artículos de revisión publicados en inglés de 1966 al 2008, encontraron RM significativas sobre su eficacia que variaron de 1.90 a 3.85, dependiendo del comparador y del tiempo de evaluación. ${ }^{21}$

Varenicline es más potente que el bupropión en términos de su eficacia para dejar de fumar (cuadro III). En un estudio sobre costo-beneficio, resultó mejor que el bupropión, la TRN, el consejo médico y dejar de fumar sin ayuda. ${ }^{22}$ Los fabricantes de varenicline enviaron al National Institute for Health and Clinical Excelence (NICE) una solicitud de evaluación tecnológica de su producto. Para ello, el NICE que es parte del sistema de salud de Inglaterra, designó a un grupo de revisores externos, quienes hicieron una evaluación con base en la mejor evidencia que el solicitante puede enviar. El reporte confirmó los hallazgos previamente publicados sobre la eficacia de varenicline, aunque en las observaciones sobre las debilidades del producto resaltan que se asume que el éxito reportado es sobre un único intento de dejar de fumar. ${ }^{23}$ Asimismo, insistieron en que se trata de un medicamento que se debe prescribir como parte de un programa de apoyo conductual. Se debe iniciar el tratamiento con tabletas de $0.5 \mathrm{mg}$ a las 8:00 horas los primeros tres días de su tratamiento y $0.5 \mathrm{mg}$ cada 12 horas los siguientes cuatro días de la primera semana. En la misma caja de la primera fase viene el tratamiento para la segunda semana que consiste en tabletas de $1 \mathrm{mg}$ para que se tomen cada 12 horas. Este esquema se recomienda completarlo por un total de 12 semanas.

\section{Tratamiento farmacológico combinado}

El cuadro III muestra el éxito obtenido utilizando la combinación de dos o más fármacos. El éxito es predecible si uno asume que la suma de dos productos que en forma individual son de utilidad en el proceso. En un metaanálisis, ${ }^{24}$ la combinación de parches con otros productos considerados de primera línea de tratamiento ofrecen más ventajas. La eficacia de las combinaciones se resume en el cuadro III. Es de notar que la combinación de goma de mascar más parches es mejor que el uso individual de goma de mascar, pero no supera al uso individual de parches. En cambio, la combinación de parches más bupropión es superior al uso individual de los dos. Se requiere más investigación para combinar. Aunque la combinación de varenicline-bupropión se usa en forma anecdótica en individuos con alto consumo e historial depresivo, se requiere investigación en este sentido

\section{Terapia conductual}

Hemos definido como conductuales todas aquellas intervenciones que no incluyen medicamentos y éstos a su vez se definieron como cualquier forma de terapia de reemplazo de nicotina, antidepresivos o varenicline o cualquier otro producto conocido como auxiliar para dejar de fumar.

Existen diferentes abordajes conductuales como ayuda para dejar de fumar. En términos generales, se pueden dividir como terapias individuales o grupales. Definimos como individuales aquellas en las que, aunque es posible que participen más personas, la intervención se puede llevar a cabo con una sola. Las individuales varían desde el consejo médico hasta abordaje con hipnosis y el uso de internet. La ventaja del conocido "consejo médico" es que su impacto es alto y puede ser dado por el médico u otro profesional de la salud.

\section{Cuadro III \\ MetaAnálisis de Terapia combinada}

\begin{tabular}{lcccccc} 
Combinación & Número de estudios & Referencia & Tiempo de evaluación & RM & IC 95\% \\
Parche + chicle & 5 & Shah $^{24}$ & 12 meses & 1.58 & $1.20-1.99$ \\
\hline Parche + bupropión & 3 & Shah $^{24}$ & 12 meses & 2.50 & $1.90-3.40$
\end{tabular}

RM: Razón de momios estimado versus placebo; IC: Intervalo de confianza. Se muestra un metaanálisis para la combinación correspondiente 
La hipnosis y la sugestión se han utilizado con éxito variable que no se ha podido registrar apropiadamente a través de ensayos clínicos controlados. ${ }^{25}$ Sin embargo, debido a que no se requieren intervenciones farmacológicas, se ha utilizado en mujeres embarazadas que no pueden dejar de fumar. ${ }^{26}$

El ejercicio se ha propuesto como una herramienta adicional, pero su utilidad tampoco se ha podido registrar a través de ensayos clínicos controlados. ${ }^{27}$ Existen reportes sobre el apoyo "en línea" (internet) cuyo análisis agrupado sugiere que solamente las intervenciones dirigidas a fumadores motivados para dejar de fumar son efectivas (RR 1.3; IC95\%; 1-1.7), 28 pero en general se requiere más investigación en esta dirección. Con respeto a la acupuntura, una revisión reciente ${ }^{29}$ sugiere que por su efecto en la liberación de algunos neurotransmisores, pudiera ser de utilidad. Sin embargo, en la Guías Clínicas Prácticas de Fiore, ${ }^{11}$ no se le otorga ninguna utilidad. En las mismas Guías aparecen una serie de abordajes conductuales como tabaquismo aversivo y rápido, apoyo social, sentimientos negativos, etcétera, cuyo tipo de evidencia es calificada como B. No las abordaremos, debido a que todas ellas requieren infraestructura, recursos humanos y el tipo de evidencia ofrecida en la actualidad no las hace prioritarias. En cambio, el consejo médico y la terapia grupal, que parecen ser muy efectivas, serán abordadas con más amplitud.
Terapia grupal

Encontramos 15 publicaciones con la búsqueda de "Group behaviour therapy", sólo nueve de ellas publicadas de 2005 en adelante y los de Cochcrane ${ }^{30}$ son los más actualizados, todos ellos aparecen en el cuadro IV, donde se resume la eficacia de la terapia grupal, comparándola con la individual y la farmacólogica. Esta modalidad es con la que nosotros, en particular, tenemos más experiencia. En nuestra clínica esta modalidad es dirigida por una psicóloga que actúa como psicoterapeuta. Conduce a un grupo máximo de 10 fumadores que quieren dejar de fumar y un mínimo de seis para conseguir la interacción dinámica. Es multimodal porque interviene un médico especialista en enfermedades respiratorias para dar consejo y consultas adicionales; además, participa una nutrióloga y se les hacen una serie de estudios para diagnóstico oportuno de varias enfermedades. El programa consiste en 10 sesiones de 90 minutos de duración, que se reparten en días diferentes durante un mes. Este programa tiene un éxito de $35 \%$ al año de observación sin grupo control ${ }^{31}$ y de $30 \%$ utilizando a pacientes con EPOC como controles. ${ }^{32}$ En la reciente revisión de Stead y Lancaster ${ }^{30}$ se encontró mayor efecto del programa grupal que la autoayuda (RM 2.04; IC95\%; 1.60-2.60), pero no encontraron diferencias con el consejo individual de la misma intensidad. No obstante, una

\section{Cuadro IV}

\section{TRATAMIENTO INDIVIDUAL VERSUS TRATAMIENTO GRUPAL Y FARMACOLÓGICO}

\begin{tabular}{|c|c|c|c|}
\hline & $\begin{array}{l}\text { Tratamiento individual } \\
\text { Consejo Médico }\end{array}$ & $\begin{array}{l}\text { Tratamiento grupal } \\
\text { Terapia psicológica }\end{array}$ & Terapia farmacológica* \\
\hline Tasa anual de abstinencia estimada \% & 12.7 & 35 & 33.2 \\
\hline RM estimado versus placebo (IC95\%) & $1.3(1.0-1.06)$ & 2.04 ( 1.60 to 2.60$)$ & $3.1(2.5-3.8)$ \\
\hline Duración del tratamiento & 3 a 10 minutos & 2 meses & 12 semanas \\
\hline Costos dólares & 16 & 84 & 450 \\
\hline Efectos adversos & Ninguno & Ninguno & Sí \\
\hline Necesidad de personal capacitado & + & +++ & ++ \\
\hline Cobertura anual $\left.\right|^{\S}$ & 42483300 & 113 & \\
\hline Cantidad aproximada de ex fumadores al año & 5097996 & 30 & \\
\hline
\end{tabular}


reciente revisión por Gordon y colaboradores ${ }^{33}$ sugiere un costo-eficiencia a esta intervención.

\section{Terapia individual}

Esta modalidad será discutida en realidad como consejo médico, aunque es claro, como se verá adelante, que no es indispensable que sea dado por un médico para que tenga impacto sobre el proceso de ayuda para dejar de fumar. La mayor parte de la información del consejo se ha hecho con médicos. En las Guías de Fiore ${ }^{11}$ se reporta una RM de 1.3 (IC95\%; 1.1-1.6), con un índice estimado de abstinencia de $10.2 \%$. En la fecha que estas Guías se publicaron, había poca información sobre el impacto que tiene el consejo de otros profesionales de la salud. En este artículo, nosotros encontramos 31 trabajos con la búsqueda "Advice to quit smoking" y tres revisiones, una relacionada con el impacto que tienen los terapistas físicos, ${ }^{34}$ otra de los médicos ${ }^{35}$ y una más de las enfermeras. ${ }^{36}$ En las tres parece haber una ventaja parecida a la que se observa con los médicos. La intervención puede ser mínima si dura menos de tres minutos, de baja intensidad si dura de 3 a 10 minutos y de intensidad más alta si dura más de 10 minutos. Las RM calculadas son de 1.3 (IC95\%; 1.01-1.6), 1.6 (IC95\%; 1.2-2.0) y 2.3 (IC95\%;2.0 -2.7), respectivamente. El tiempo de contacto en cada sesión es proporcional a la efectividad de la intervención. El mayor impacto se consigue cuando el contacto es de 31 a 90 minutos. Asimismo, las evidencias, resumidas en las Guías de Fiore ${ }^{11}$ señalan que más de ocho sesiones incrementan la probabilidad de dejar de fumar en $25 \%$, en comparación a darle una sesión o ninguna (RM 2.3; IC95\%; 2.1-3.0).

El consejo médico es la herramienta disponible más simple con la que el médico, y en general el personal de salud, cuenta para influir de manera decisiva en un fumador, para que deje de fumar, aumentando la motivación y/o el cambio de estadio en el fumador para pasar, ${ }^{37}$ por ejemplo, de precontemplador a contemplador. Este consejo puede lograr más de 5\% de exfumadores al año. Una medida de calidad en un hospital es el interés de los médicos por la anamnesis del tabaco y el consejo en los pacientes fumadores. Desafortunadamente sólo $2 \%$ de los pacientes que dejan de fumar han sido aconsejados por el médico. ${ }^{38}$

En resumen, las evidencias señalan que la participación del médico u otros profesionales de la salud para motivar al fumador a que deje de fumar, incrementa las probabilidades de éxito hasta 2.5 veces más, en comparación con no hacer nada cuando se atiende a pacientes fumadores. Los fumadores consideran el consejo médico o la recomendación para dejar de fumar una motivación importante.

\section{Abordaje multimodal}

Si bien la combinación de dos o más fármacos es una alternativa que funciona mejor que una sola, agregar a esta suma de medicamentos la terapia conductual es posible que incremente más el éxito en el tratamiento. En una revisión se discute esta posibilidad. ${ }^{39}$ En la práctica, este abordaje multimodal es común, sin embargo, no hay estudios que se hayan dirigido específicamente a comparar un abordaje multimodal versus monoterapía. Se requiere más investigación en este sentido.

\section{Discusión}

La manera de entender el fenómeno mundial del tabaquismo, su crecimiento y su distribución es crucial para poder controlarlo. El tabaquismo es un fenómeno cerebral bioquímico como recientemente se ha demostrado, que resulta, entre otros factores, de la mercadotecnia. Por lo tanto, se requiere tener un enfoque amplio, que tome en cuenta la experiencia y el conocimiento de diversos especialistas, tanto de las ciencias médico-biológicas como de las sociales y económicas, así como de los aspectos económicos de cada región. Por ejemplo, los costos anuales de atención médica atribuibles al consumo de tabaco en Latinoamérica oscilan entre 6 y 14\% de los gastos personales en salud, derivando en altos costos de atención médica y representa un alto costo social por las muertes prematuras que genera en la sociedad. Lo anterior deriva en un empobrecimiento de la sociedad en su conjunto, efecto que se ve más pronunciado en los países en desarrollo, especialmente en los estratos más desfavorecidos. ${ }^{40}$ Esta arista debe considerarse en cualquier propuesta o guía para el tratamiento del tabaquismo.

Aunque se ha ido aclarando que hay una serie de factores hereditarios y neurofarmacológicos que influyen en la respuesta terapéutica de la adicción a la nicotina y otras drogas, el tratamiento debe incluir el abordaje directo y personalizado. Asimismo, desde el punto de vista de las políticas de salud, se debe considerar seriamente la disponibilidad real que tiene el gobierno para suministrar sin costo los medicamentos e intervenciones que el fumador necesita. El trabajo de Raw y colaboradores ${ }^{10}$ muestra que independientemente de que no todos los países cuentan con todos los medicamentos recomendados por la FDA para el tratamiento del tabaquismo, sólo $26 \%$ o menos recibe reembolso parcial de su aseguradora por la compra de auxiliares para dejar de fumar, como TRN, bupropión, varenicline o terapia conductual, y sólo en 22\% de los países participantes la ayuda está disponible fácilmente como parte de los medicamentos en los servicios públicos de salud. 
En México, por ejemplo, prácticamente ninguno de los productos considerados de primera línea está disponible fácilmente en las instancias de asistencia médica y seguridad social pública como el Instituto Mexicano del Seguro Social o el Instituto de Seguridad y Servicios Sociales de los Trabajadores del Estado, sistemas de salud que no son gratuitos, sino que resultan del pago de una cuota que se deduce a un trabajador. Aunque la TRN existe en el cuadro básico (lista mínima de medicamentos), en los hechos es poco probable que haya y que se le prescriba a un fumador derechohabiente. Lo mismo ocurre para bupropión y varenicline, que con certeza no están incluidas en el cuadro básico. En relación con el sector conocido como de la Secretaría de Salud, no hay disponibilidad de estos medicamentos. Por lo tanto, se requiere utilizar el recurso que sí está disponible, el humano.

En este trabajo hemos realizado una revisión de las guías existentes y lo que en esencia recomiendan a la población a la que están dirigidas para tratar el tabaquismo. Aunque parece claro que el uso de los medicamentos conocidos como de primera línea son los primeros que deben recomendarse, no queda claro en qué orden debe ocurrir esta recomendación. Por otro lado, las guías tampoco consideran la realidad económica de cada país y, de hecho, se concretan a exhibir el éxito de cada intervención y prácticamente queda a la decisión del profesional por cual intervención empezar. La única intervención que queda clara en todas las guías y tiene un nivel de evidencia $\mathrm{A}$, es el consejo médico. En este sentido, independientemente de la disponibilidad de medicamentos, el consejo médico y la terapia grupal pueden aplicarse en todos los países.

De ahí que una propuesta deguía en el tratamiento del tabaquismo en los países como México y Latinoamérica, en los que a pesar de la disponibilidad comercial de toda clase de medicamentos auxiliares para dejar de fumar, no existe la disponibilidad pública (subsidio por el estado o los sistemas públicos de salud), se debe contemplar lo siguiente:

a) tratamiento conductual individualizado (consejo médico) y

b) tratamiento conductual grupal (a través de clínicas de ayuda para dejar de fumar).

Ya hemos mostrado el impacto que tienen tanto el consejo médico como la terapia grupal. La pregunta siguiente será cuál es la mejor opción. En el cuadro V hacemos un ejercicio estimativo del número de fumadores que serían abordados por un médico, así como el número de ellos que dejaría de fumar. El cuadro parece indicar que incluso en el plano más pesimista, es decir, que un médico atendiera solamente a un fumador a la semana, el número de fumadores que dejaría de fumar es atractivo para cualquier programa de salud pública. Por otro lado, sabemos que los fumadores que consumen más de 20 cigarros al día son los que con más dificultad dejan de fumar. En ellos, es probable que el consejo médico sea insuficiente y requieran el apoyo de clínicas especializadas. Por lo tanto, se requieren acciones concretas que hemos desglosado brevemente en los siguientes párrafos.

\section{Formación y capacitación de recursos para el tratamiento individualizado por medio del consejo médico}

Se ha observado que la participación del médico u otros profesionales de la salud para motivar al fumador a que

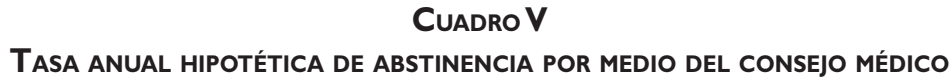

\begin{tabular}{|c|c|c|c|c|}
\hline Número de médicos & Consulta por semana* & Consulta por mes $\stackrel{\ddagger}{\ddagger}$ & Consulta anual $\S$ & Numero de ex fumadores al año ${ }^{t}$ \\
\hline I & 6 & 30 & 300 & 36 \\
\hline 10 & 60 & 300 & 3000 & 360 \\
\hline 100 & 600 & 3000 & 30000 & 3600 \\
\hline 1000 & 6000 & 30000 & 300000 & 36000 \\
\hline 100000 & 60000 & 300000 & 3000000 & 360000 \\
\hline
\end{tabular}

* El 70\% de la consulta vista por un médico son pacientes fumadores. Esta estimación se hizo con la hipótesis de que cada médico atendiera a nueve pacientes por semana, seis de ellos serían fumadores

¥ Tomando cinco días hábiles

$\S$ Calculado a 10 meses por días feriados y vacaciones

${ }^{t}$ Calculando una tasa anual de abstinencia de $12 \%$ 
deje de fumar incrementa las probabilidades de éxito hasta 2.5 veces más en comparación con no hacer nada. Cuando se atiende a pacientes fumadores, los fumadores consideran el consejo médico o la recomendación para dejar de fumar una motivación importante. Cuando se emplea un consejo breve de al menos tres minutos, resulta en un incremento en la tasa de abstinencia comparado con no intervención (RM 1.3). Existe una relación dosis-respuesta en el tiempo empleado para el consejo, incrementando al doble las tasas de éxito cuando se emplean más de 10 minutos en comparación con tres minutos (por ejemplo, se logra una tasa de abstinencia de 22.1\% (RM 2.3; IC95\%; 2.0-2.7) para intensidad alta y $13.4 \%$ (RM 1.3; IC95\%; 1.0-1.06) para un consejo mínimo. ${ }^{38}$

Cualquier país que capacite a todo el personal médico, paramédico o promotor de la salud en el ejercicio del consejo médico como una practica cotidiana de su quehacer profesional y que integren el tema del tabaquismo como el "nuevo signo vital", como recientemente Hays y colaboradores ${ }^{38}$ han propuesto, daría un paso gigante en el control del tabaquismo. Realizando una estimación hipotética, ese país obtendría muchos éxitos logrando que un número considerable de fumadores dejara de fumar. Por ejemplo, en México, según cifras del INEGI, ${ }^{41}$ en 2006 había 141611 médicos en la República mexicana y en promedio atendieron a dos pacientes fumadores por día. Esto representó un registro de 3540275 consultas mensuales y 42483300 anuales. Si cada médico diera el consejo médico a sus pacientes de por lo menos tres minutos, y tomando una tasa de éxito anual de $12 \%$ en un año, se obtendrían 5097996 exfumadores. Considerando que la prevalencia de fumadores en 2006 fue de 14 millones, ${ }^{42}$ se requerirían tres años para obtener una tasa de abstinencia de $100 \%$ en pacientes que acuden al médico por cualquier motivo. Por lo tanto, en países con escasos recursos económicos como la mayoría de los países de Latinoamérica, el tratamiento individual y personalizado que se logra por medio del consejo breve sería una estrategia adecuada para el control del tabaquismo. Sin embargo, menos de $50 \%$ de los médicos investigan el consumo de tabaco en el paciente o en algún familiar durante la consulta general.

En México, el antecedente de tabaquismo está considerado dentro de los antecedentes patológicos del expediente clínico, es decir, que, por el sólo hecho de ser fumador, el individuo ya es considerado portador de una enfermedad, lo que implica que debe ser atendido. La recomendación de considerar al consejo médico como un nuevo signo vital, significa que al mismo tiempo que se determina la presión arterial, el pulso y la temperatura corporal de un paciente, se determine también el ante- cedente pasado o actual de tabaquismo. Así, el médico podrá abordar al paciente con un consejo para que deje de fumar en ese momento o minutos después. Este consejo debe ser claro, positivo, personalizado, adecuado e intensivo. Actualmente existe un algoritmo fácil de recordar conocido como las " 5 aes". La propuesta es que cada vez que un médico en el primer nivel de atención se ponga en contacto con un paciente, ya sea fumador o no, aplique la regla de las Aes. Es decir Averigüe, Aconseje, Analice, Apoye y Arregle. Una revisión detallada de las "5 Aes", se puede encontrar en las Guías de Fiore. ${ }^{11}$

\section{Creación, capacitación y certificación de clínicas de ayuda para dejar de fumar}

Durante los últimos 10 años se ha fomentado la creación de clínicas de ayuda para dejar de fumar, que estén al alcance de todos los individuos, familias y comunidades con base en criterios de calidad y calidez, logrando costos que sean accesibles para toda la población. ${ }^{43} \mathrm{Su}$ propósito fundamental es apoyar a todos los individuos interesados en dejar de fumar y disminuir los daños en su salud ocasionados por el tabaquismo. Dependiendo de su capacidad de atención y respuesta ofrecidas, estas clínicas se distinguen por niveles de atención, recordando que cada clínica y su respectivo nivel es un concepto funcional más que estructural o económico. Es decir, se aspira a que, por ejemplo, todos los consultorios médicos del país sean potencialmente clínicas de ayuda para dejar de fumar, correspondiente al primer nivel de atención. La aplicación del segundo y tercer nivel de atención asume la existencia de unidades hospitalarias con capacidad para ofrecer servicios de prevención secundaria y terciaria. Asimismo, estos centros tienen la capacidad de ofrecer tratamiento y rehabilitación, a través de consulta externa y/o de hospitalización. Sin embargo, este esfuerzo requiere un sistema de vigilancia y capacitación continua para el adecuado funcionamiento y desarrollo de las clínicas.

El cuadro IV muestra las ventajas y desventajas comparativas de la terapia grupal a través de clínicas versus el consejo médico. Es claro que éste último parece tener más ventajas. Sin embargo, siempre debe existir la opción del abordaje multimodal desde donde se puede ofrecer terapia conductual y farmacológica. Aunque esto puede hacerse en el consultorio médico, el tiempo es insuficiente. Por lo tanto, las clínicas tienen un enorme campo de acción.

En resumen, hemos presentado una propuesta de tratamiento integral poniendo al consejo médico, ofrecido por cualquier profesional de la salud y a la terapia grupal a través de clínicas de ayuda para dejar de fumar, como los ejes desde los cuales se puede ofrecer no sola- 
mente el apoyo conductual, sino el farmacológico. Las opciones que existen de intervenciones farmacológicas se revisaron y se discutieron y la conclusión es que se cuenta con un vasto arsenal con el que el trabajador de salud puede contar.

\section{Declaración de conflicto de intereses}

Declaramos no tener conflicto de intereses.

\section{Referencias}

I. Pérez-Bautista O, Ramírez-Venegas A, Escobar-Arriaga E, Sansores RH. Differences in inflammatory markers in a non-smoking and smoking Mexican population. Rev Invest Clin 2009;61:205-II.

2. Ezzati M, López AD. Estimates of global mortality attributable to smoking in 2000. Lancet 2003;362:847-52.

3. Ezzati M, Henley SJ, Thun MJ, López AD. Role of smoking in global and regional cardiovascular mortality. Circulation. 2005; I 2:489-97.

4. Menezes AM, Lopez MV, Hallal PC, Muiño A, Pérez-Padilla R, Jardim $J R$, et al and the PLATINO Team. Prevalence of smoking and incidence of initiation in the Latin American adult population: the PLATINO study. BMC Public Health 2009;9:151.

5. Sansores RH, Ramírez-Venegas. Guías Mexicanas para el tratamiento del tabaquismo. Un consenso nacional de expertos. Neumología y Cirugía de Tórax 2005;64(S2):S84-S109.

6. Müller F, Webe L. Smoking and smoking cessation in Latin America: a review of the current situation and available treatments. Int ] Chron Obstruct Pulmon Dis 2008;3(2):285-293.

7. Slama, K. Prevención y control del tabaquismo. Guía para países con escasos recursos económicos. International Union Against Tuberculosis and Lung Disease Paris, 2000.

8. Reichert J,Araújo A, Cantarino Goncalvez CM, Godoy I, Chatkin JM, Uchoa M, et al. Smoking Cessation Guidelines $2008 \mathrm{~J}$ Bras Pneumol 2008;34(I0):845-880.

9. Global Initiative for Chronic Obstructive Lung Disease. Global Strategy for the Diagnosis, Management, and Prevention of Chronic Obstructive Pulmonary Disease. Updated 2009. [consultado 2010 enero 18] Disponible en: http://www.goldcopd.com/Guidelineitem. asp? ||$=2 \&|2=|$ \&int $\mid d=2003$.

10. Raw M, Regan S, Rigotti N, Mc Neill A. A survey of tobacco dependence treatment services in 36 countries.Adicction 2009;104(2):279-287.

I I. Fiore MC, Jaén CR, Baker TB, Bailey WC, Benowitz NL, Curry SJ, et al.Treating tobacco use and dependence: 2008 update US Public Health Service Clinical Practice Guideline executive summary. Respir Care 2008;53(9) I217-I 222.

12. Moore D,Aveyard P, Connock M, Wang D, Fry-Smith A, Barton $P$. Effectiveness and safety of nicotine replacement therapy assisted reduction to stop smoking: Systematic review and meta-analysis. BMJ 2009;338:bl024.

|3. Hughes JR, Stead LF, Lancaster T.Antidepressants for smoking cessation. Cochrane Database Syst Rev 2007; I:CD00003I.

14. Eisenberg MJ, Filion KB, Yavin D, Bélisle P, Motillo S, Joseph L, et al. Pharmacotherapies for smoking cessation: a meta-analysis of randomized controlled trials. CMAJ 2008; 179(2):135-44.
15. Wu P,Wilson K, Dimoulas P, Mills EJ. Effectiveness of smoking cessation therapies: a systematic review and meta-analysis. BMC Public Health 2006;6:300.

16. Demyttenaere K, Jaspers L. Bupropion and SSRI-induced side effects. J Psychopharmacology 2008;22:792-804.

17. Nides M. Update on Pharmacologic Options for Smokings Cessation Treatment. Am J Med 2008;12I(4):S20-S3I.

18. Boshier A, Wilton LV, Shakir SA. Evaluation of the safety of bupropion (Zyban) for smoking cessation from experience gained in general practice use in England in 2000. Eur J Clin Pharm 2003;59:767-73.

19. Cahill K, Stead LF, Lancaster T. Nicotine receptor partial Agonists for smoking cessation. Cochrane Database Syst Rev 2007; I6(I):CD006103. 20. Cahill K, Stead LF, Lancaster T. Nicotine receptor partial Agonists for smoking cessation. Cochrane Database Syst Rev 2008; I6(3):CD006103. 21. Garrison GD, Dugan SE.Varenicline: a first-line treatment option for smoking cessation. Clin Ther 2009;3I (3):463-49I.

22.Annemans L, Nackaerts K, Bartsch P, Prignot J, Marbaix S. Cost effectiveness of varenicline in Belgium, compared with bupropion, nicotine replacement therapy, brief counselling and unaided smoking cessation: a BENESCO Markov cost-effectiveness analysis. Clin Drug Investig 2009:29:655-65.

23. Hind D, Tappenden P, Peters J, Kenjegalieva K.Varenicline in the management of smoking cessation: a single technology appraisal. Health Technol Assess 2009;13(S2):9-13.

24. Shah SD, Wilken LA, Winkler SR, Lin SJ. Systematic review and metaanalysis of combination therapy for smoking cessation. J Am Pharm Assoc 2008;48:659-665.

25. Covino NA, Bottari M. Hypnosis, behavioral theory, and smoking cessation. J Dent Educ 200 I;65(4):340-7.

26. Barron J, Petrilli F, Strath L, McCaffrey R. Successful interventions for smoking cessation in pregnancy.Am J Matern Child Nurs 2007;32(I):48-49. 27. Grimshaw GM, Stanton A. Tobacco cessation interventions for young people. Cochrane Database Syst Rev 2006;(4):CD003289.

28. Shahab L, McEwen A. Online support for smoking cessation:a systematic review of the literature. Addiction 2009; 104: 1792-1804. 29. Cabioglu MT, Ergene N, Tan U. Smoking cessation after acupuncture treatment. Int J Neurosci 2007; I I7(5):57I-578.

30. Stead LF, Lancaster T. Group behaviour therapy programmes for smoking cessation. Cochrane Database Syst Rev 2005;(2):CD00I007. 31. Méndez Guerra M, Urdapilleta E, Sansores RH, Lara Rivas G, RamírezVenegas A, Regalado-Pineda J, et al. Factores que determinan que un paciente ingrese a un programa para dejar de fumar. Rev Inst Nal Enf Resp Mex 2009;22(I):7-13.

32. Flores-Trujillo F, Ramírez-Venegas A, Hernández-Zenteno R, Méndez Guerra M, Sansores RH. Comparación del éxito para dejar de fumar a largo plazo en sujetos con EPOC y sin EPOC. Rev Inst Nal Enf Resp Mex 2009;22(I):21-28.

33. Gordon L, Graves N, Hawkes A, Eakin E.A review of the costeffectiveness of face-to-face behavioural interventions for smoking, physical activity, diet and alcohol. Chronic IIIn 2007;3(2):10I-129. 34. Bodner ME, Dean E. Advice as a smoking cessation strategy: a systematic review and implications for physical therapists. Physiother Theory Pract 2009;25:369-407.

35. Stead LF, Bergson G, Lancaster T. Physician advice for smoking cessation. Cochrane Database Syst Rev 2008;(2):CD000I65. 36. Rice VH, Stead LF. Nursing interventions for smoking cessation. Cochrane Database Syst Rev 2008;(I):CD00I 188.

37. Stead LF, Lancaster T. Interventions to reduce harm from continued tobacco use. Cochrane Database Syst Rev 2007;(3):CD00523I. 38. Hays JT, Ebber JO, Sood A. Treating Tobacco Dependence in Light of the 2008 US Department of Health and Human Services Clinical Practice Guideline. Mayo Clin Proc 2009;84(8):730-736. 
39. Reus VI, Smith BJ. Multimodal techniques for smoking cessation: a review of their efficacy and utilization and clinical practice guidelines. Int J Clin Pract 2008;62(II):1753-1768.

40. Reynales-Shigematsu LM. Costos de atención médica de las enfermedades atribuibles al consumo de tabaco en América: revisión de la literatura. Salud Publica Mex 2006;48(suppl I):S190-S200.

41. Instituto Nacional de Estadística, Geografía e Informática. Encuesta Nacional de la Dinámica Demográfica 2006 (ENADID 2006). México: INEGI, 2006.
42. Kuri-Morales PA, González-Roldán JF, Hoy MJ, Cortés-Ramírez M. Epidemiología del tabaquismo en México. Salud Publica Mex 2006;48(supl I):S9I-S98.

43. Rubio H, Sansores RH, Oviedo MT, Espinosa M,Vélez BA, Castrejón V. Modelo para la prestación de servicios en materia de tabaquismo en México. Rev Ins Nal Enf Resp Mex 2003;16:92-102.

44. Stead LF, Perera R, Bullen C, Mant D, Lancaster T. Nicotine replacement therapy for smoking cessation. Cochrane Database Syst Rev 2008;(I):CD000I 46. 